\title{
058
}

\section{ANEMIA SIDEROPENICA E PRESENZA DI HELICOBACTER PYLORI NELLE FECI}

Grassi P., Grasso E., Torrisi C., Guardo G., Scrifignano V., Trapanotto G., Patamia I., Sciacca A, Nicoletti G.

Laboratorio Analisi Azienda Policlinico Università Catania

Nell'ultimo decennio le conoscenze sul rapporto patologie umane ed Helicobacter pylori si sono evolute ed ampliate. Sono stati, inoltre, messi a punto una grande varietà di test invasivi e di laboratorio che ha permesso di facilitarne la diagnosi.

La ricerca diretta degli antigeni di Helicobacter pylori nelle feci consente di evidenziare l'infezione indipendentemente dalla localizzazione gastrica ma soprattutto permette un largo uso nei bambini anche molto piccoli e la possibilità di verificare i risultati di una terapia eradicante dopo breve tempo.

L' antigene Helicobacter pylori è stato ricercato, con metodo E.L.I.S.A., su 450 campioni di feci. I campioni fecali provenivano da piccoli pazienti la cui età era compresa tra 2-15 anni con sintomatologie varie: anemia sideropenica (70\%) piastrinopenia, scarso accrescimento, patologie intestinali (dolori, vomito, dispepsia, bruciori), malattie autoimmuni (tiroidite, celiachia, artriti autoimmuni)

Il $30 \%$ dei pazienti con anemia sideropenica e piastrinopenia era risultato positivo per Helicobacter pylori, mentre nei piccoli con disturbi gastro-enterici la positività si riscontrava solo nel $10 \%$ dei casi .

Il contagio familiare è stato confermato in questa casistica, ben 9 nuclei familiari osservati presentavano positività all'antigene di Helicobacter pylori nelle feci

Il trattamento antibiotico mirato per Helicobacter pylori è stato risolutivo in 11 pazienti con anemia sideropenica; infatti la stabile normalizzazione dei depositi marziali è stata ottenuta solo dopo terapia eradicante per Helicobacter pylori e senza alcuna somministrazione di composti a base di ferro.

L'elenco delle malattie correlato alle infezioni sostenute da Helicobacter pylori tende ad aumentare ma sono soprattutto le prospettive di ricerca che sembrano sconfinare in campi fino a pochi anni fa inimmaginabili. 